\title{
HIGH PERFORMANCE CARBON NANOTUBE/SILICON HETEROJUNCTION SOLAR CELLS
}

\author{
Xiaokai Li ${ }^{l}$, Yeonwoong Jung ${ }^{2}$, Yong Sun ${ }^{2}$, Mark A. Reed ${ }^{2}$ and André D. Taylor ${ }^{1 *}$ \\ ${ }^{1}$ Chemical \& Environmental Engineering Department \\ ${ }^{2}$ Electrical Engineering Department \\ Yale University, New Haven, CT 06511 USA
}

\begin{abstract}
A key obstacle for Terra-watt deployment of solar cells is the high cost of materials and fabrication processes. Here, we report the development of an advanced spraying technique for the fabrication of carbon nanotube (CNT) and crystalline silicon ( $\mathrm{Si}$ ) heterojunction solar cells for both planar and dual radial geometries. The nanostructured dual radial p-n junction array solar cells offer a great opportunity to produce efficient solar cells with low cost materials. We have optimized the spray technique and engineered the $\mathrm{CNT} / \mathrm{Si}$ interface for $\mathrm{CNT} / \mathrm{Si}$ solar cell of planar geometry and achieved power conversion efficiency (PCE) of $8.5 \%$ at $\mathrm{AM} 1.5 \mathrm{G}$. For $\mathrm{CNT} / \mathrm{Si}$ solar cell of dual radial geometry, initial test revealed an ideality factor of 3.85 , which suggests that these devices can be further optimized beyond this initial proof of concept.
\end{abstract}

\section{INTRODUCTION}

Photovoltaic (PV) solar cells are attractive candidates for clean and renewable power. Although significant advancements have been made in the energy conversion efficiency, the normalized energy costs of PVs still remain higher than using traditional fossil fuels [1]. Nanostructured radial p-n junction arrays offer a great opportunity to produce efficient solar cells, by decoupling light absorption and charge separation. This unique architecture facilitates improved light scattering and trapping using low cost materials. Previously both Si nanowire (SiNW) (Fig. 1a) [2] and nanopore (Fig. 1b) [3] radial p-n junction architectures have been explored. The cells showed performance in SiNWs and nanopore structures superior to that in planar ones, demonstrating a promising power conversion efficiency (PCE) of 11\% [4]. However, these geometry cells required a series of complicated fabrication processes with some steps at elevated $\left(1000{ }^{\circ} \mathrm{C}\right)$ temperature.

Recent research efforts toward high-efficiency solar cells have been driven by the use of novel materials with superior materials properties coupled with low cost fabrication processes. In this regard, CNT/Si heterojunction solar cells satisfy both these criteria with the following advantages: (1) CNTs possess excellent electronic (e.g., high carrier mobility and reduced scattering), structural (e.g., low density and mechanical flexibility), and optical (e.g., wide range of direct band gaps and strong photo-absorption) properties, suitable for photovoltaic components. (2) CNTs can be processed by wet chemical methods at low temperature and are easy to extrinsically $p$-dope (e.g., acid treatments) for controlling photovoltaic properties. (3) heterojunction is formed via a simple/inexpensive solution casting or mechanical transfer of CNTs onto planar Si substrate, which is also compatible with well-established Si-based photovoltaics, presently the dominant technology of the market. (4) CNT/Si solar cells are less susceptible to air/environment-sensitive degradation, which CNT-organic solar cells generally suffer from.

Here we show a new approach that exploits the radial junction of $\mathrm{Si}$ hollow pillar arrays with doped single walled carbon nanotubes. Remarkably, no radial heterojunction devices with

CNT and Si have been reported to date due to technological challenges of conformal wrapping $\mathrm{Si}$ pillars or pores with nanometer scale thin CNT films. Previously reported CNT/Si devices only have the CNT sitting on the top of the pillars leading to low efficiency of $1.29 \%$ [5]. Our results reveal that this new architecture is viable and can be further improved using several processing steps that we previously developed for multifunctional composite materials [6].
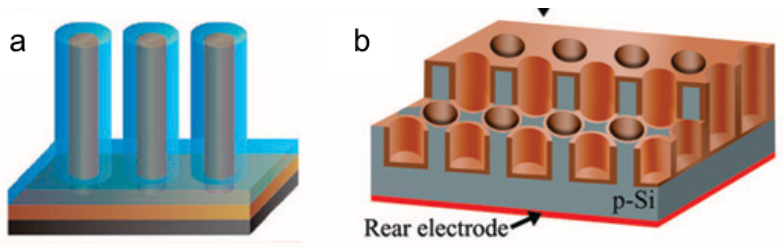

Figure 1. (a) Schematic cell design with the single crystalline n-Si $N W$ core in brown, the polycrystalline $p$-Si shell in blue. (b) Si nanohole solar cell with radial $p$ - $n$ junctions via thermal phosphorus dopant diffusion. The $n+$ layer is shown in purple bronze, the p-Si substrate in gray. (Figure 1 a and $b$ are adapted from reference [1] and [2] respectively.)

\section{RESULTS AND DISCUSSION}

We developed a low temperature spraying technique that allows the CNTs to wrap around and inside the hollow Si pillars (Fig. 2) forming a CNT/Si dual radial heterojunction (Fig. 3).

a
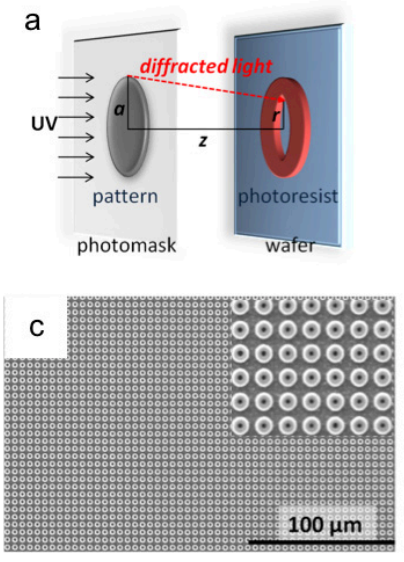

Figure 2. Hollow Si pillar fabrication procedure. (a) Schematic to illustrate the principle of diffraction lithography. (b) Schematic to illustrate the experimental set-up. (c) SEM image to show a large-area uniformity of photoresist rings. (d) SEM image to show an array of vertically aligned hollow Si pillars.

Previously, CNTs have been sprayed on flat Si wafers to make CNT/Si solar cells of flat geometry with a 4\% PCE [7]. Using our advanced spraying techniques, we demonstrate an $8.5 \%$ 
PCE at AM1.5 (Fig. 4a). Based on this success, we have optimized the hollow pillar structures and increased the interfacial area $(10 \mathrm{X})$ over the flat geometry $(10 \mu \mathrm{m}$ tall, $0.3 \mu \mathrm{m}$ and $1 \mu \mathrm{m}$ inner and outer diameter respectively with $1 \mu \mathrm{m}$ spacing). Initial results show an ideality factor of 3.68 (Fig. 4b).

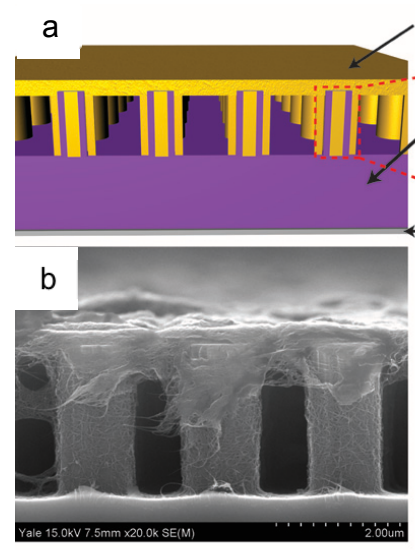

Side View
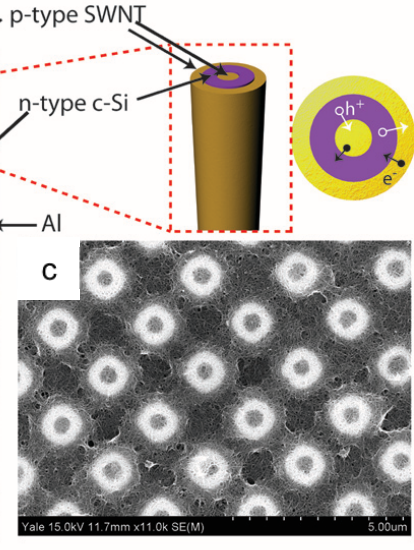

Top View
Figure 3. CNT/ Si dual radial heterojunction solar cell. (a) illustration of the heterojuntion and SEM picture of (b) side view and (c) top view.

At present, the ideality factor (3.68 in Fig. 4b) of the dual radial CNT/Si solar cell is not as high as that (1.95 in Fig. 4a) of the planar CNT/Si solar cell. This high ideality factor suggests that the interface states of heterojunctions need to be further improved since the bulk properties of used CNT and Si are identical. The CNT in the dual radial geometry cell is interfaced with top-down dry-etched $\mathrm{Si}$, which inherently possesses large defects on the surface, while the planar cell is made of CNT interfaced with the pristine, single-crystalline Si surface. Un-optimized interfaces lead to large carrier recombination, short carrier lifetime, and thus a high ideality factor. Efforts are presently being made to optimize the interfacial properties toward suppressed carrier recombination, e.g., better adhesion between $\mathrm{CNT}$ and $\mathrm{Si}$, improving the surface crystallinities of $\mathrm{Si}$ tubes by utilizing various surface passivation/etching techniques. Nevertheless, one noticeable advantage of this dual radial heterojunction solar cell is its large optical absorption due to greatly increased interfacial areas over either conventional planar cells or dual radial heterojunction cells made of solid pillars without inner holes. This benefit is believed to be significant enough to compensate for the high ideality factor, thus leading to a higher overall efficiency.

We can control the feature sizes of vertically aligned hollow Si pillars using our recently developed single step diffraction optical lithography [8]. (Fig. 2) This simple and well-controlled method relies on the diffraction of UV light from opaque patterns on a photomask, and utilizes the central diffraction maximum, which forms behind the patterns. This single-step lithographic approach doesn't require any extra cost over conventional optical lithographies since the hollowness of the photoresist is formed through a spontaneous diffraction effect, which is also highly controllable without any need for extra experimental apparatus. Moreover, this method can fabricate sub-wavelength features on a large-scale, e.g., the radial thickness of our $\mathrm{Si}$ tubes are down below $\sim 100 \mathrm{~nm}$ which are $<\sim 1 / 4$ of the used wavelength, which conventional optical lithographies can hardly achieve. Other non-optical (e.g., e-beam lithography) or alternative optical (e.g., interference) lithographies capable of sub-wavelength feature fabrication are generally very costly and require a series of fabrication process and experimental apparatus, unlike ours.
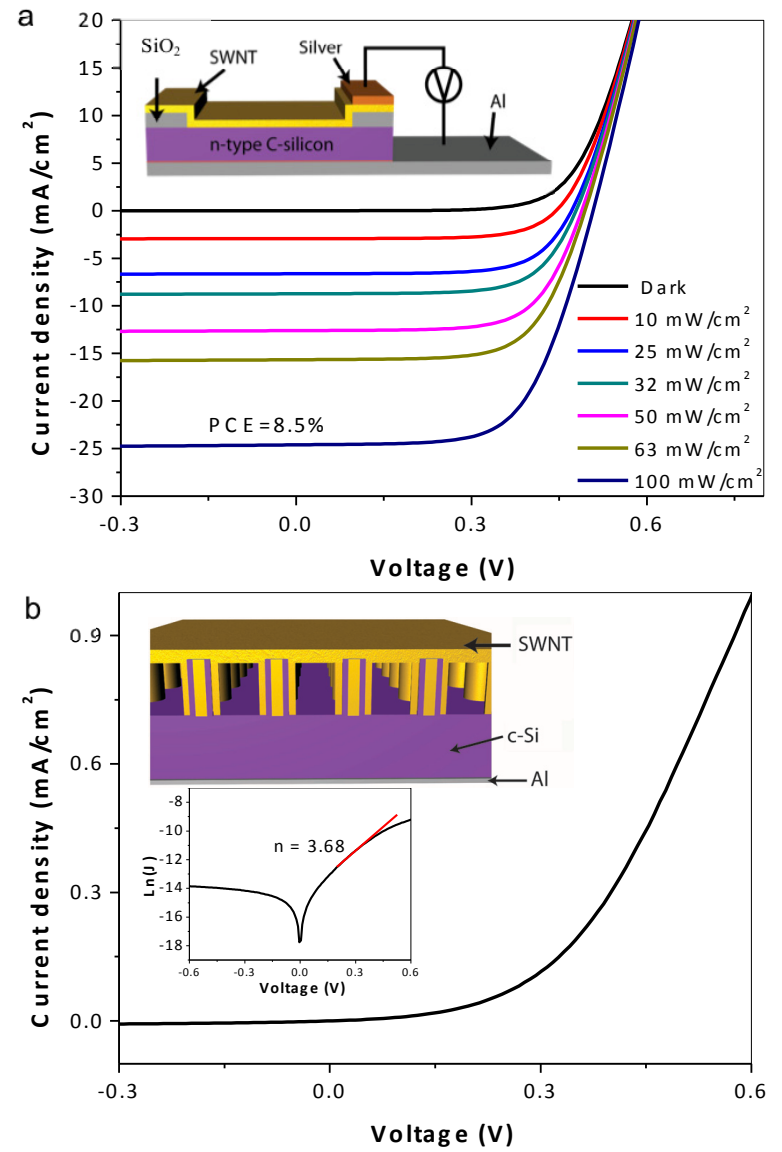

Figure 4. CNT/Si heterojunction solar cell. (a) J-V curve of flat heterojunction. The inset illustrates the flat heterojuntion. The ideality factor of the device is 1.95. (b) Dark J-V for CNT/Si dual radial heterojuntion. The ideality factor of the device is 3.68.

Fig. 2a illustrates the experimental arrangement for the case of diffraction from the circular disk on a transparent photomask with an accurately controlled separation $\mathrm{z}$ introduced between the photomask and a photoresist-coated wafer. Consequently, light diffracted behind the disk $\mathrm{z}$ is selectively chosen to expose the photoresist, forming a bright spot in the center of the shadow of the disk. Fig. $2 b$ shows the schematic experimental set-up where the separation $\mathrm{z}$ was accomplished by an intentionally created photoresist spacer attached to the photomask. Fig. 2c illustrates an SEM image of a large-area array of photoresist rings. Fig. 2d shows the resultant hollow Si pillar array after reactive ion etching process.

\section{CONCLUSIONS}

In summary, we have described an ordered dual radial heterojunction structure from a low temperature solution process, which promises higher light absorption, better carrier separation and collection, and higher solar cell efficiency.

\section{ACKNOWLEDGEMENTS}

X.L. and A.D.T. acknowledge financial support by the SOLAR program of the National Science Foundation under DMR-0934520. 


\section{REFERENCES}

[1] N.S. Lewis, "Toward Cost-Effective Solar Energy Use", Science, 798, 315 (2007).

[2] E.C. Garnett, P. Yang, "Silicon Nanowire Radial P-N Junction Solar Cells", Journal of the American Chemical Society, 9224, 130 (2008).

[3] K.Q. Peng, X. Wang, L. Li, X.L. Wu, S.T. Lee, "High-Performance Silicon Nanohole Solar Cells", Journal of the American Chemical Society, 6872, 132 (2010).

[4] D.R. Kim, C.H. Lee, P.M. Rao, et al., "Hybrid Si Microwire and Planar Solar Cells: Passivation and Characterization", Nano Letters, 2704, 11 (2011).

[5] Q. Shu, J. Wei, K. Wang, et al., "Hybrid Heterojunction and Photoelectrochemistry Solar Cell Based on Silicon Nanowires and Double-Walled Carbon Nanotubes", Nano Letters, 4338, 9 (2009).
[6] X. Li, F. Gittleson, M. Carmo, R.C. Sekol, A.D. Taylor, "Scalable Fabrication of Multifunctional Freestanding Carbon Nanotube/Polymer Composite Thin Films for Energy Conversion", ACS Nano, 1347, 6 (2012).

[7] Z. Li, V.P. Kunets, V. Saini, et al., "Light-Harvesting Using High Density p-type Single Wall Carbon Nanotube/n-type Silicon Heterojunctions", Acs Nano, 1407, 3 (2009).

[8] Y. Jung, A. Vacic, Y. Sun, E. Hadjimichael, M. Reed,"Mapping of Near Field Light and Fabrication of Complex Nanopatterns by Diffraction Lithography", Nanotechnology, 045301, 23 (2012).

\section{CONTACT}

*André D. Taylor, tel: (203) 432-2217; andre.taylor@yale.edu 